\title{
New response functions for absorption-line indices from high-resolution spectra
}

\author{
R. Tantalo ${ }^{1}$, C. Chiosi ${ }^{1}$ and L. Piovan ${ }^{1}$ \\ ${ }^{1}$ Department of Astronomy, University of Padova, Vicolo dell'Osservatorio, 335122 \\ Padova, Italy \\ email: rosaria.tantalo@unipd.it
}

\begin{abstract}
Using a huge library of 1- $\AA$ resolution spectra over a wide range of $\log T_{\text {eff }}, \log g$ and $[\mathrm{Fe} / \mathrm{H}]$ and for both solar and $\alpha$-enhanced abundance ratios $[\alpha / \mathrm{Fe}]$, we derive the response functions $\left(\mathcal{R} \mathcal{F}_{\mathrm{S}}\right)$ which are commonly used to correct indices with solar $\alpha$-enhanced ratios to indices with $\alpha$-enhanced $>0$. The $\mathcal{R} \mathcal{F}_{\mathrm{S}}$ vary not only with the type of star but also with metallicity. With the aid of this and the fitting functions $\left(\mathcal{F} \mathcal{F}_{\mathrm{S}}\right)$, we derive the indices for single stellar populations. The new indices for single stellar populations are used to derive the age, metallicity, and degree of enhancement first for sample of Galactic globular clusters for which these key parameters have been independently derived from the colour-magnitude diagram and/or spectroscopic studies, and second to study the classical two index diagram.
\end{abstract}

Keywords. galaxies: evolution, galaxies: abundances

\section{New response functions and SSP indices}

To derive the response functions $\left(\mathcal{R} \mathcal{F}_{\mathrm{S}}\right)$ adopted in this work we made use of the partial pre-release of the synthetic spectral library by Munari et al. (2005) at 1- $\AA$ resolution,degraded to the Lick resolution, for the following parameters: two values of $[\alpha / \mathrm{Fe}]=0$ and +0.4 dex; six values of $\left[\mathrm{Z} / \mathrm{Z}_{\odot}\right]$, i.e. $-2.0,-1.5,-1.0,-0.5,0$, and 0.5 ; five values of $T_{\text {eff }}$, i.e. $4000,5250,7250,10000$, and $13000 \mathrm{~K}$; finally eleven values of $\log g$ going from 0 to 5.0 in steps of 0.5 . This library requires the enhancemnt definition at varing $\mathrm{Z}\left(\Gamma_{\alpha}\right)$ (see for all details Tantalo et al. 2007)

We calculate the new $(\mathcal{R} \mathcal{F} \mathrm{S})$ making the differences: $(\delta I)_{T_{\text {eff }}, g,\left[\mathrm{Z} / \mathrm{Z}_{\odot}\right]}=I_{\text {enh }}-I_{\text {sol }}$ taken at fixed $\log T_{\text {eff }}, \log g$, and metallicity $\left(\left[\mathrm{Z} / \mathrm{Z}_{\odot}\right]\right)$ and varying $\Gamma_{\alpha}$ from 0 to 0.4 , whose corresponding indices are indicated as $I_{\text {sol }}$ and $I_{\text {enh }}$, respectively.

The indices for a solar-scaled mixture are calculated as described in Tantalo \& Chiosi (2004b), whereas those for $\Gamma_{\alpha}>0$ are obtained according to the following equation: $I_{i, \text { enh }}=I_{i, \text { sol }}+\delta \mathcal{I}_{i}$, where $I_{i \text {,sol }}$ is the solar-scaled index of the generic star in the SSP and $I_{i, \text { enh }}$ the same but corrected for enhancement by $\delta \mathcal{I}_{i}$.

\section{Comparison with observations}

Globular Cluster. It is worth checking whether the present indices can reproduce the data for the globular clusters. To do so we took the sample of Galactic globular clusters and Bulge compiled by Puzia et al. (2002) for which indices in the Lick system have been measured. The results are shown in Fig. 1, the overall agreement is satisfactory.

To further confirm the quality of the theoretical indices, we derive the ages, metallicities, and enhancement degree from the indices for the sub-set of this sample of globular cluster for which the estimate of ages and metallicities (i.e. $[\mathrm{Fe} / \mathrm{H}]$ ) have been derived from the colour-magnitude diagrams (see Table 1), and consider the current estimates of 


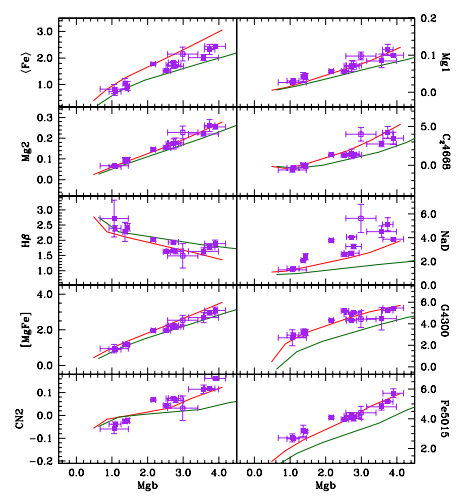

Figure 1. The Mgb index versus ten different indices calculated with the metallicities typical of globular clusters. The thin-solid lines are the models with solar-scaled pattern of abundances, whereas the thick-solid are the models with $\Gamma_{\alpha}=0.4$. Along each line, indices of SSPs with the same age $(\sim 12 \mathrm{Gyr})$ are shown. The metallicity increases along each line from $\left[\mathrm{Z} / \mathrm{Z}_{\odot}\right]=-2$ ( $\mathrm{Z} 0.0001)$ to 0 (Z 0.019), moving from left to right. The data are from Puzia et al. (2002).

Table 1. Ages, metallicities and $[\alpha / \mathrm{Fe}]$ for the sample of Galactic Globular Clusters by Puzia et al. (2002). Columns (2) through (5) are estimates of the parameters based on the colour-magnitude diagrams or spectroscopic measurements; columns (6) through (8) are the results derived from the absorption line indices.

\begin{tabular}{c|cccc|ccc}
\hline $\mathrm{NGC}$ & {$[\mathrm{Fe} / \mathrm{H}]_{C G}$} & {$[\alpha / \mathrm{Fe}]^{d}$} & $\mathrm{~T}_{C G}$ & $\sigma_{T}$ & {$[\mathrm{Z} / \mathrm{H}]$} & {$[\alpha / \mathrm{Fe}]$} & Age \\
\hline 5927 & -0.64 & - & 10.0 & 0.7 & -0.62 & 0.34 & 12.6 \\
6218 & -1.14 & 0.35 & 10.0 & 0.9 & -1.76 & 0.30 & 7.9 \\
6284 & -1.13 & - & 9.5 & 0.4 & -1.51 & 0.37 & 8.9 \\
6356 & -0.89 & - & 10.0 & 2.0 & -0.94 & 0.37 & 12.6 \\
6388 & -0.74 & - & 10.6 & 2.0 & -0.91 & 0.06 & 10.0 \\
6528 & 0.07 & 0.11 & 10.0 & 2.0 & -0.03 & 0.16 & 12.6 \\
6553 & -0.06 & 0.19 & 10.0 & 2.0 & -0.03 & 0.03 & 12.6 \\
6624 & -0.70 & - & 10.6 & 1.4 & -0.91 & 0.30 & 12.6 \\
6637 & -0.78 & - & 9.9 & 1.1 & -1.03 & 0.37 & 12.6 \\
6981 & -1.21 & - & 8.7 & 0.9 & -1.74 & 0.34 & 10.0 \\
\hline
\end{tabular}

enhancement (i.e. $[\alpha / \mathrm{Fe}]$ ), obtained directly from stars, as indicators of the total degree of enhancement in each cluster. To derive the theoretical parameters, we consider the different sensitivity of the indices to each parameter and we apply the so-called recursive minimum-distance method $\dagger$ described in Tantalo et al. (2007). The results of this analysis are shown in right part of Table 1.

Early-type galaxies. The two-indices diagnostic planes, $H \beta$ versus $[\mathrm{MgFe}]$ plane shown in the left panel of Fig. 2, are often used to infer the age, metallicity and degree of enhancement (see Tantalo \& Chiosi 2004a).

Considering that the age of the Universe provided by WMAP is $13.7 \pm 0.2 \mathrm{Gyr}$, there is a significant number of galaxies falling below the $13 \mathrm{Gyr}$ age-line even for the solar-scaled compositions. One of the reasons could be the insufficient contamination by emission in particular for $H \beta$ (it worth recalling that the data have been corrected for emission). The emission, not included in our theoretical indices, is by far more uncertain to quantify. To

$\dagger$ This method was first introduced and applied in the first version of the paper by Tantalo \& Chiosi (2004a) appeared on http://xxx.lanl.gov/abs/astro-ph/0305247v1 Section 10 to which the reader should refer for details. However, it was dropped from the final version of the paper to shorten the text. 

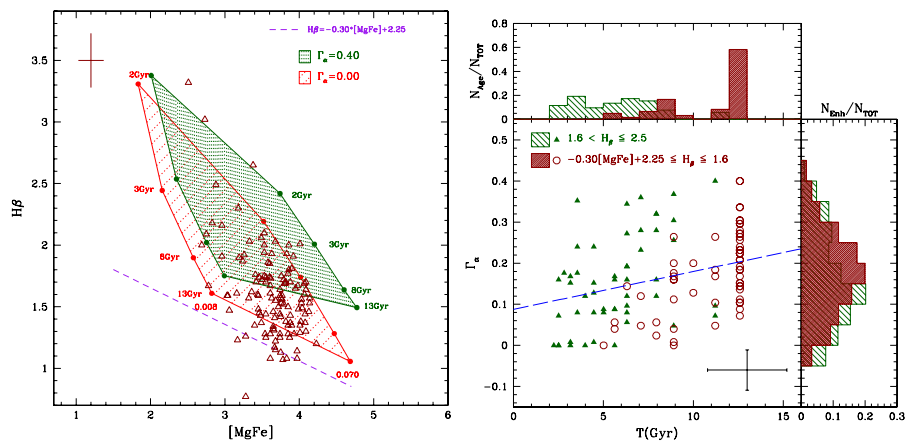

Figure 2. Left Panel: $H \beta$ versus [MgFe] plane. Comparison between theory and observational data for galaxies in the local universe. Each hatched area is comprised between two SSPs of different metallicity $(\mathrm{Z}=0.008$ left-side and $\mathrm{Z}=0.070$ right-side). Along each SSP the age increases from 2 (top) to $13 \mathrm{Gyr}$ (bottom). Each area is for a different $\Gamma_{\alpha}$ as indicated. The data are the normal galaxies (open-triangles) of Trager et al. (1998) corrected for emission (mean error bars are indicated). The dashed line is meant to provide a lower limit to $H \beta$ below which observational uncertainties may be so strong to render the comparison with theory meaningless (see the text for more details). Right Panel: $\Gamma_{\alpha}$ versus $\mathrm{T}$ (in Gyr) plane. The data correspond to the sub-sample of Trager catalogue selected to have $-0.30[\mathrm{MgFe}]+2.25 \leqslant H \beta \leqslant 2.5$. Open circles are for $-0.30[\mathrm{MgFe}]+2.25 \leqslant H \beta \leqslant 1.6$ and filled triangles for $1.6<H \beta \leqslant 2.5$. Long-dashed lines show the linear best fit for the whole sample. Mean error in age and $\Gamma_{\alpha}$ is shown. The top panel gives the age distribution for both sub-sample as indicated, whereas the right panel gives the $\Gamma_{\alpha}$ distribution.

reconcile things the correction already applied by Trager et al. (1998) should be increased to about $0.2 \AA$. So we prefer to drop from the sample all galaxies whose $H \beta$ falls below a suitable limit which is represented by dashed line shown in left panel of Fig. 2.

The large scatter in $H \beta$ shown by galaxies in this panel is generally attributed to a scatter in ages. Stands on our results, the dependence of $H \beta$ on $\Gamma_{\alpha}$ makes this conclusion questionable at least for galaxies whose $H \beta$ falls in the range 1 to $1.6-1.8$. Leaving aside galaxies with very strong $H \beta(\geqslant 1.8)$ for which the age scatter seems to be unavoidable), let us concentrate on the region where $H \beta$ is in the range 1 to 1.6. In this interval, models predict that $H \beta$ increases as function of $\Gamma_{\alpha}$ by a factor $\Delta H \beta=0.2$ to about 0.4 at increasing metallicity. Therefore, the effect of $\Gamma_{\alpha}$ on $H \beta$ in particular, let us to predict that among old objects of the same age there could be a natural scatter in the diagnostic planes caused by galaxy to galaxy variations in the mean degree of enhancement due to the particular star formation history (Tantalo \& Chiosi 2004b)

To cast light on this issue the following analysis is performed. We start selecting from the sample of galaxies a sub-set suited to our purposes: first we drop all galaxies with $H \beta$ larger than 2.5 as they are very likely affected by ongoing or recent star formation; secondly we drop all galaxies whose $H \beta$ is below the dashed line shown in the left panel of Fig. 2. The remaining galaxies should represent a fair sub-sample. We need now to specify what we mean by normal- and strong- $H \beta$. As the range spanned by $H \beta$ for variations in $\mathrm{Z}, \Gamma_{\alpha}$ and age (we assume $1 / 3$ of the Hubble time, i.e. $4 \mathrm{Gyr}$ ) is of the order of $0.3 \AA$, we add this quantity to the mean value for a solar scaled old galaxy $(\sim 1.4 \AA)$ and take $H \beta=1.6$ as the transition from typical to high values.

Using the ages, metallicities and $\Gamma_{\alpha}$ found with the minimum-distance method for this sub-sample of galaxies, in the right panel of Fig. 2 we correlate the age with $\Gamma_{\alpha}$ using different symbols for galaxies with low- and high- $H \beta$. Galaxies with $H \beta \leqslant 1.6$ (empty circles) are preferentially ( 66 per cent) old $(13 \mathrm{Gyr})$ and spanning a large range of $\Gamma_{\alpha}$; in this group, however, there are also a few younger objects with $\Gamma_{\alpha} \leqslant 0.2$. Galaxies with 
$H \beta>1.6$ tend to be younger (71 per cent between 2 and $7 \mathrm{Gyr}$ ) (more recent episodes of star formation) and less enhanced in $\alpha$-elements ( $\sim 67$ per cent with $\left.\Gamma_{\alpha} \leqslant 0.2\right)$, and to span a narrower range of $\Gamma_{\alpha}$ than in the previous group. However also in this case there are objects of both old age and high $\Gamma_{\alpha}$. The age and $\Gamma_{\alpha}$ distributions for the two groups are shown in the top and right panels in the right part of Fig. 2.

Finally, taking the linear best fit of the data regardless of $H \beta$ (long-dashed line) the general trend emerges: at increasing age the degree of enhancement increases as shown by the long dashed line in the central panel right-hand of Fig. 2.

\section{Conclusions}

We have generated synthetic absorption-line indices on the Lick system based on the recent library of $1-\AA$ resolution spectra calculated by Munari et al. (2005) over a large range of atmospheric parameters $\left(\log T_{\text {eff }}, \log g\right.$ and $\left.[\mathrm{Fe} / \mathrm{H}]\right)$ and both for solar and $\alpha$ enhanced abundance ratios in the chemical composition. The main results of this study are:

(i) First we derive a modern version of the so-called $\mathcal{R} \mathcal{F}_{\text {s. }}$. Contrary to the previous situation in which the $\mathcal{R} \mathcal{F}_{\mathrm{S}}$ were known only for three stars of given $\log T_{\text {eff }}$ and $\log g$, now the $\mathcal{R} \mathcal{F}_{\mathrm{S}}$ are given for large range ranges of $\log T_{\text {eff }}, \log g$ and $[\mathrm{Fe} / \mathrm{H}]$ (or $\left[\mathrm{Z} / \mathrm{Z}_{\odot}\right]$ ). Not only the $\mathcal{R} \mathcal{F}_{\mathrm{S}}$ vary with the type of star but also with the metallicity. The effect of metallicity is important and cannot be neglected.

(ii) With the aid of the new $\mathcal{R} \mathcal{F}_{\mathrm{S}}$ and the fitting functions $\left(\mathcal{F} \mathcal{F}_{\mathrm{S}}\right)$ indices for SSPs are calculated. The present results clearly demonstrate that not only all indices depend on the enhancement but also that $H \beta$ increases with it as already anticipated by Tantalo \& Chiosi (2004a).

(iii) The present results lend support to the suggestion made by Tantalo \& Chiosi (2004b) that in the $H \beta$ versus [MgFe] plane (and similar) galaxies with $1<H \beta<1.6$, whose scatter in $H \beta$ is customarily due to age (and metallicity), could be indeed old objects of the same age (say $13 \mathrm{Gyr}$ ) that scatter in $H \beta$ because of differences in metallicity and enhancement from galaxy to galaxy likely caused by their individual star formation history. Only occasionally, especially for the strong $H \beta$ galaxies, more recent episodes of star formation ought to be invoked. Using the theoretical estimates of the three parameters we look for general correlations and find that at increasing age of the last episode of star formation the mean enhancement increases in agreement with current understanding of supernova explosions on galactic chemical enrichment.

\section{References}

Munari, U., Sordo, R., Castelli, F., \& Zwitter, T. 2005 A $\& A$ 442, 1127

Puzia, T., Saglia, R., Kissler-Patig, M., Maraston, C., Greggio, L., Renzini, A., \& Ortolani, S. 2002, A\&A 395,45

Tantalo, R. \& Chiosi, C. 2004a MNRAS 353, 917

Tantalo, R. \& Chiosi, C. 2004b MNRAS 353, 405

Tantalo, R., Chiosi, C., \& Piovan, L. 2007, A\&SA 462, 481

Trager, S.C., Worthey, G., Faber, S.M., Burstein, D., \& González, J.J. 1998 ApJS 116, 1 